\title{
Estrategia para usar racionalmente el agua: experiencia de una entidad de educación técnica industrial, certificada bajo la norma ISO 14001:2004
}

\section{RESUMEN}

En la costa del Perú, región que concentra al $60 \%$ de la población y en donde se ejerce una alta presión sobre el recurso hídrico, resulta beneficioso para el Servicio Nacional de Adiestramiento en Trabajo Industrial (SENATI), contar con una estrategia para usar racionalmente el agua. Para ello, estableció para su sede principal, ubicada en el distrito de Independencia, tres ejes para mejorar el uso del agua: Definir mecanismos de control, usar tecnologías adecuadas y mantener una conciencia ambiental en los usuarios. Estos ejes le permitieron reducir malas prácticas a razón del $20 \%$ en el primer año de implementación. Desde entonces y hasta ahora, ha resultado fundamental trabajar en concientizar a los usuarios sobre la importancia del uso racional del agua, con la finalidad de seguir manteniendo ratios promedio de $0,35 \mathrm{~m}^{3} /$ persona/año.

Palabras clave: agua, estrategia ambiental, uso racional

STRATEGY TO USE THE WATER RATIONALLY:

EXPERIENCE IN A TECHNICAL SCHOOL, CERTIFIED UNDER ISO 14001: 2004

\section{ABSTRAC}

On the coast of Peru, a region that concentrates $60 \%$ of the population and where a very high pressure on water resources exist, is beneficial to the National Service for Industrial Training (SENATI), have a strategy to use the water. To do this, set for its headquarters, located in the district of Independence, three axes to improve water use: Define control mechanisms, using appropriate technologies and maintain an environmental awareness among users. These axes allowed it to reduce wastes at the rate of $20 \%$ in the first year of implementation. From then until now, has been instrumental work in raising awareness among users about the importance of rational use of water, in order to continue to maintain average rates of $0,35 \mathrm{~m}^{3} /$ person/year.

Keywords: environmental strategy, rational use water

\section{INTRODUCCIÓN}

En nuestro país, especialmente en la región costera y específicamente en la ciudad de Lima, es baja la percepción de las personas sobre la importancia que tiene la disponibilidad del recurso hídrico en todas aquellas actividades desarrolladas de manera cotidiana. El agua es utilizada en muchas ocasiones de manera poco racional. Las empresas industriales suelen registrar sus costos de producción y uno de ellos suele ser el uso del agua en sus procesos productivos, sin embargo el bajo costo del metro cúbico hace que prácticamente sea un costo insignificante respecto a otros costos asociados como la mano de obra, insumos o incluso la energía (eléctrica o calorífica).

En función de lo anterior y en oposición a ello, aparece entonces la necesidad de encontrar otra motivación para utilizar el agua de manera racional, es aquí donde se incorpora el concepto "medioambiental" o comportamiento "ecológico" de distintas organizaciones. Entre ellas, aquellas entidades que han implementado o certificado un sistema de gestión ambiental basado en el estándar internacional ISO 14001:2004, la cual es una norma voluntaria que establece requisitos para constituir un sistema de gestión orientado a prevenir la contaminación. En relación a la implementación de una norma como la ISO 14001:2004, dicha decisión se toma al más alto nivel dentro de cualquier organización que decida hacerlo, es por ello que normalmente se encuentra dentro del marco del planeamiento estratégico.

Dentro del proceso de implementación de los requisitos de ISO 14001:2004 está aquel vinculado al establecimiento de "Objetivos, metas y programas" (Requisito 4.3.3) relacionado a su vez a la "Identificación de aspectos ambientales" (Requisito 4.3.1) que para el tema central del presente artículo está vinculado al "Uso del agua".

El uso del agua en el SENATI es un aspecto ambiental significativo, el cual a su vez cuenta con un objetivo asociado desde el año 2006. Vinculado a ello, fue que estratégicamente se establecieron tres frentes para apoyar la consecución de la meta establecida: la determinación de mecanismos de control (políticas / lineamientos / Instrucciones), el uso de tecnologías apropiadas y la concientización de los usuarios del agua, a todo nivel (alumnos, personal propio, tercero y visitantes).

\footnotetext{
Ingeniero ambiental, Universidad Nacional Agraria la Molina, gerente general Cercanna S.A.C.
} E-mail: theotorres@cercanna.com 
En este sentido, se presenta como ejemplo de aplicación de una estrategia apropiada para utilizar el agua de manera racional, aquella utilizada en el SENATI, específicamente en su sede institucional más grande y de mayor afluencia de personas, ubicada en el distrito de Independencia, Lima, Perú.

\section{BASES TEÓRICAS}

\subsection{El recurso hídrico en el Perú}

Según la Autoridad Nacional del Agua (ANA), el Perú está catalogado como el segundo país con mayores recursos hídricos a nivel mundial; sin embargo gran parte de la población carece de este recurso vital para su subsistencia. La razón principal es la falta de infraestructura que permita una justa y racional distribución, en especial en la región costera, donde se concentra el $60 \%$ de la población nacional.

El agua es un recurso vital, presente e indispensable en toda actividad humana. Su disponibilidad, propicia condiciones higiénicas, sanitarias, de esparcimiento y recreación, de aprovechamiento directo en actividades productivas o de servicios, vivienda o espacio público.

En la actualidad, el uso del agua en organizaciones de distintos tipos y tamaños, dista mucho de ser racional. El consumo diario per cápita se estima en 50 litros en locales educacionales, según el Sistema Nacional de Estándares de Urbanismo del Ministerio de Vivienda, Construcción y Saneamiento en su propuesta preliminar de 2011. Sin embargo, la realidad indica que este consumo debe estar muy por encima del promedio estimado.

\subsection{La problemática del agua}

La relación del hombre con la naturaleza, la pobreza y las crecientes desigualdades en el orden mundial, generan un círculo vicioso en torno al tema del agua. Simultáneamente con la crisis ambiental, las poblaciones pobres de los países en desarrollo se han estancado o ido empobreciendo aún más, haciéndose más marcado las diferencias entre los Productos Brutos Internos (PBI) de los países ricos con los pobres. Así, mientras en los años 60, la diferencia de ingresos era de 30 a 1, en los 90 era de 60 a 1, según. López B. [1].

Actualmente, los países desarrollados, con $20 \%$ de la población mundial, manejan el $85 \%$ de las finanzas globales y los países en desarrollo con el $80 \%$ de la población mundial, solo representan el $22 \%$ del PBI del planeta. En los últimos 30 años, el $20 \%$ de la población que concentra la mayor riqueza en el mundo, aumentó su participación en el ingreso global de un $70 \%$ a un $85 \%$.

La reconfiguración del escenario económico mundial, conlleva una marcada y continua polarización en la distribución de la riqueza, cada vez son más los incluidos en el concepto de desarrollo, siendo también mayores las necesidades del agua, elemento básico para cualquier forma de vida y que apoya sistemas de riego, industria, minería, generación de electricidad. Es a la vez vía de transporte y comunicación y además, el principal espacio donde nos dejamos descansar. Sin embargo, está repartida de manera desigual o es utilizada de manera inadecuada dividiendo a las poblaciones en privilegiadas o carentes de dicho recurso.

El Perú cuenta con 106 cuencas hidrográficas por las que escurren 2,1 millones de metros cúbicos (MMC) de agua al año. Asimismo, cuenta con 12200 lagunas en la sierra y más de 1007 ríos, con los que se alcanza una disponibilidad media de recursos hídricos de 2,5 MMC concentrados principalmente en la vertiente amazónica (Ministerio de Agricultura, 2009). La sierra y la costa, donde habita el $90 \%$ de la población, dispone de menos de $2 \%$ de este recurso. Hecho que representa una gran presión demográfica sobre los recursos hídricos que dispone la región costera.

Sin embargo, su disponibilidad en el territorio nacional es irregular, puesto que casi el $70 \%$ de todo el agua precipitada se produce entre los meses de diciembre y marzo, teniéndose épocas de extrema aridez en todo el año y de abundante lluvia en algunos meses. Además, muchas lagunas han sufrido el impacto de la contaminación por desechos mineros, agrícolas y urbanos, y el asentamiento de pueblos en sus orillas.

Por otra parte, en la costa, región donde reside el $60 \%$ de la población, se recibe una precipitación anual de $38 \mathrm{~mm}, 16$ veces menos que la región andina y casi 100 veces menos que la región amazónica. La escasez de agua es una situación que hace un llamado a la reflexión de toda la sociedad en su conjunto.

\subsection{Estrategia ambiental}

Sobre la estrategia ambiental, podemos mencionar que se establece como un plan cuyo fin es mitigar los impactos ambientales negativos, que pudieran ser generados por las operaciones de una organización. Estos impactos ambientales incluyen aquellos relacionados con el uso y agotamiento de los recursos naturales, emisiones atmosféricas, 
vertimientos líquidos, ruidos, disposición inadecuada de residuos, entre otros.

Winn y Angell [2] indican que el análisis de la estrategia ambiental de las organizaciones puede hacerse desde tres diferentes perspectivas: Los modelos de rendimiento social, los modelos de proceso estratégico y los modelos de gestión ambiental. Los primeros consideran que la respuesta corporativa a las cuestiones ambientales es un tipo de respuesta social, de manera que está motivada por diferentes filosofías o aproximaciones de la empresa al ambiente más que por otros factores motivadores de contenido económico, normativo o ético. Desde esta perspectiva, la estrategia ambiental de la empresa comienza con la toma de conciencia por parte de la alta dirección de la necesidad de proporcionar una respuesta a los problemas ambientales.

La generalización de las consideraciones ambientales en la sociedad, al igual que otros cambios del entorno, puede alterar la posición competitiva empresarial dentro de un sector productivo, sugiriendo nuevas oportunidades y amenazas a las que cada organización debe dar una respuesta diferente, que depende tanto de sus puntos fuertes y débiles, como de la percepción de estos.

Cada actividad industrial o comercial, conlleva actividades, productos o procesos que tienen un efecto diferente sobre el ambiente (emisiones atmosféricas, residuos, vertidos, deforestación, etc.) por lo que las respuestas de la empresa a las oportunidades y amenazas ambientales serán distintas. Por tanto, para analizar la estrategia ambiental de operaciones de las empresas parece conveniente seleccionar únicamente una rama o sector económico en el que sea posible controlar estas diferencias entre organizaciones pertenecientes a industrias distintas, Handfield et al. [3].

Por otro lado, la problemática ambiental ha sido objeto de estudio fundamentalmente de las empresas manufactureras sometidas a una exigente legislación ambiental; sin embargo, las empresas de servicios, calificadas por Hutchinson [4] como las destructoras silenciosas del ambiente, han sido objeto de una menor atención, amparadas quizás en un menor impacto directo sobre el entorno.

De este modo, una estrategia ambiental requiere la evaluación completa del funcionamiento de la empresa; en el caso de los establecimientos educativos, esto incluye desde el aprovisionamiento de los factores productivos (tales como energía, agua, alimentos, productos de limpieza, materiales didácticos, etc.), pasando por la tecnología empleada (equipos y máquinas), el control de actividades y procesos (como la separación de residuos sólidos o el control del uso de recursos), hasta el mismo producto (servicios de formación y capacitación) y la eliminación de subproductos (reutilización y reciclaje de residuos, emisiones, vertimientos, etc.). Podemos pues, apuntar que una gestión ambiental adecuada no solo es responsable de la consecución del producto deseado en términos de calidad y cantidad, sino que también debe realizar un control de las prácticas de trabajo, del consumo de recursos y las emisiones generadas, así como del flujo de productos y sustancias peligrosas, según Gupta y Sharma [5].

Esta integración de las cuestiones ambientales, es una tarea compleja ya que debe conjugar el rendimiento económico y el ambiental, no olvidando que además este último presenta varias dimensiones. Ante esta complejidad, Azzone y Noci [6] señalan la conveniencia de establecer una taxonomía de estrategia ambiental de operaciones que nos permita identificar bajo qué condiciones es más conveniente cada tipo de estrategia ambiental. En este sentido, se definen a continuación las dimensiones que permiten caracterizar los distintos tipos de estrategia ambiental de operaciones aplicables a los establecimientos educativos. Para ello, siguiendo a Azzone y Noci [6] se distingue entre factores externos (la presión de los grupos de interés hacia la protección ambiental) y factores internos (como su nivel de gestión ambiental, la formalización de esa gestión ambiental, la experiencia en gestión ambiental y el dominio de las técnicas de la Dirección de Operaciones).

\section{METODOLOGÍA}

Las actividades previstas para desarrollar la estrategia para usar el agua de manera racional estuvieron previstas bajo el esquema del Ciclo de Mejora Continua, Ciclo de Deming o Ciclo PHVA:

\section{Planificación}

1. Definir el alcance de la estrategia.

2. Definir el objetivo y cuantificar la meta.

3. Establecer los mecanismos de control.

4. Identificar las tecnologías actuales disponibles para el uso del agua.

\section{Implementación}

5. Difusión de metas y de los mecanismos de control establecidos. 
6. Incrementar el grado de conciencia ambiental de los usuarios del agua.

7. Revisión y/o cambio de tecnologías para el uso del agua (riego de áreas verdes, servicios higiénicos, comedores, etc.).

\section{Verificar}

8. Cuantificar y reportar los consumos de agua en función de un ratio.

9. Consolidar el reporte de ratios de consumos de agua.

\section{Actuar}

10. Retroalimentar a las sedes involucradas.

11. Brindar los recursos necesarios para cambio de tecnologías para el uso del agua.

\subsection{Fuentes de Información}

El presente artículo, basa sus resultados en las distintas fuentes consultadas, tales como: La documentación facilitada por la entidad educativa, políticas y lineamientos, registros y estadísticas que muestran el uso del agua a través del tiempo, su evolución y comportamiento.

La información vinculada a la segmentación de los principales actores involucrados en esta investigación, se obtuvo de la Dirección Académica, así como directamente de las personas a través de las encuestas. Se analizaron los resultados de las encuestas aplicadas a los distintos actores involucrados en el quehacer diario de la entidad educativa, en su Sede Independencia, la cual incluye también el edificio de la Dirección Nacional.

\subsection{Población en estudio}

Conociendo las características de la población a estudiar en la entidad educativa, se consideró adecuado utilizar el muestreo probabilístico, de tipo aleatorio y estratificado, en el cual se seleccionaron cuotas de individuos con características similares. Grupos representativos de los trabajadores de la entidad educativa, tales como personal docente, administrativo y de terceros como los agentes de seguridad, limpieza, jardinería, servicios varios y comedor. También, están los alumnos que representan a la población más numerosa.

En la sede Independencia, se tienen aproximadamente 1000 trabajadores y se estima que diariamente asisten entre 3500 y 4500 alumnos. Se consideró entonces un promedio de 4000 alumnos. Por tanto, el promedio de personas que al día están en la sede serían 5000.
La estrategia para el uso racional del agua implementó lo siguiente:

\subsection{Mecanismos de control}

Los mecanismos de control establecidos en la institución educativa, se basan en el establecimiento de objetivos de "uso racional" del agua, para ello se establecen planes que contemplan las siguientes acciones: Programas de mantenimiento preventivo de instalaciones sanitarias, renovación de griferías y sanitarios cuyas características incluyan ahorro del agua, detección y reporte de fugas y la concientización de los usuarios.

\subsection{Tecnologías aplicadas}

Las tecnologías utilizadas para el uso racional del agua en el SENATI, están directamente relacionadas con el tipo de actividades propias de una entidad educativa. Entre las actividades más importantes tenemos: Riego de áreas verdes, servicios higiénicos, servicios de alimentación, limpieza, entre otros.

Los resultados se obtuvieron a través de:

\subsection{Medición de los consumos (ratios)}

Desde el año 2006, una de las medidas iniciales aplicadas por la entidad educativa fue medir la cantidad de agua utilizada en todo el predio de la sede Independencia, tal como se muestra en la siguiente gráfica:

Como se aprecia en la Gráfica 1, el consumo identificado inicialmente en el año 2006 fue de más de $100000 \mathrm{~m}^{3} / a n ̃ o$, luego de la aplicación de varias medidas (de gestión, tecnológicas y de concientización) fue que se pudo optimizar el consumo en aproximadamente $20 \%$ y $21 \%$ para los años 2007 y 2008 respectivamente, en relación al año base (2006). Fue en estos dos años que se trabajó principalmente en el tema culturar de los usuarios, es decir, minimizando malas prácticas a través de la concientización y sensibilización respecto al recurso agua.

En los años siguientes, el efecto de las medidas no se aprecia significativamente debido a que fue en estos años que el SENATI experimentó un crecimiento considerable respecto al número de alumnos y trabajadores. Es por ello que el consumo totalizado de agua en la Sede Independencia ha ido creciendo desde aproximadamente $100000 \mathrm{~m}^{3}$ en el año 2006 hasta los $160000 \mathrm{~m}^{3}$ consumidos en el 2013 (No se referenció al año 2014, porque no se tuvo el dato del pago por consumo de agua). Así también, es importante mencionar que desde 
Gráfica 1. Consumo y pago anualizado del agua en la Sede Independencia

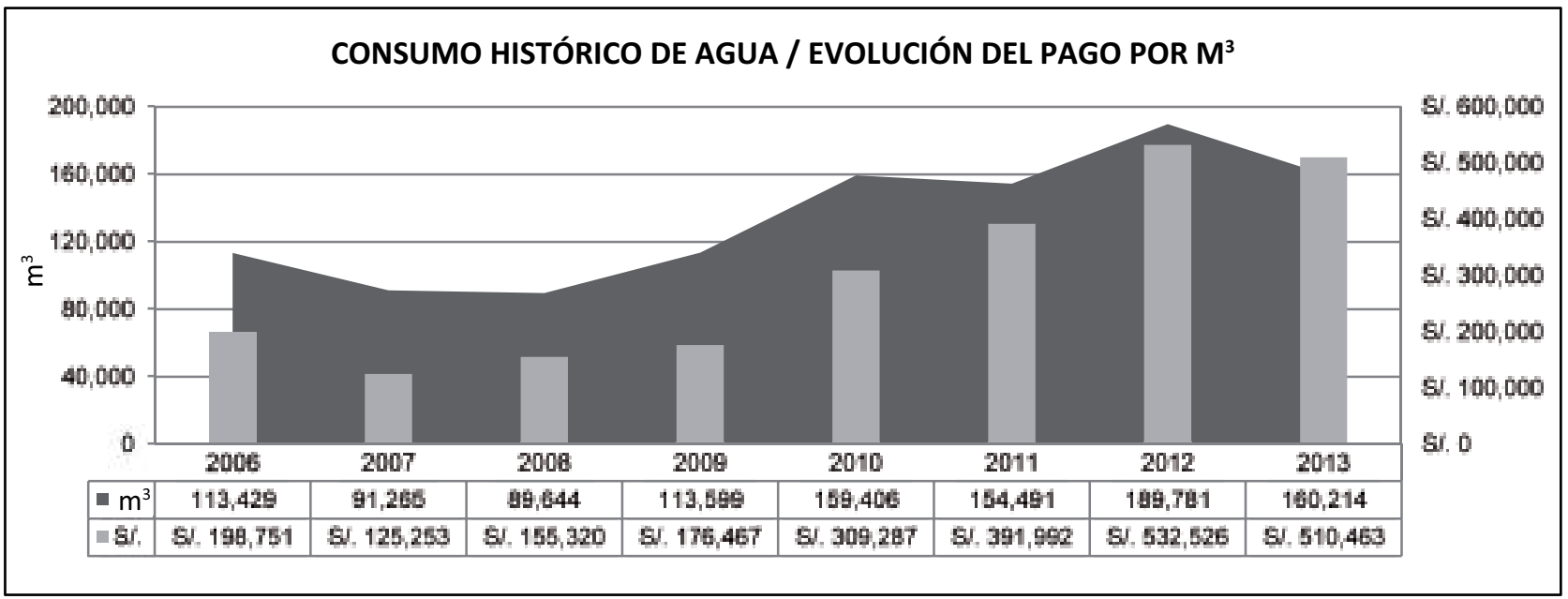

Fuente: Sistema Integrado de Gestión - SIG del SENATI.

el año 2006 el monto anual pagado por agua era aproximadamente 200000 soles, pasando a más de 500000 soles, lo cual equivale a un incremento de 2.5 veces el monto pagado desde el 2006.

En este sentido, es importante resaltar que adicionalmente a ser una iniciativa ambiental, el uso racional del agua es un tema que genera un interés económico para cualquier administración, dado que en solo 7 años el precio pagado por metro cúbico se incrementó 2.5 veces. Sin embargo, es una realidad que el número de usuarios también se ha ido incrementando anualmente, tanto alumnos como personal de la entidad educativa y personal contratista, por lo que el ratio encontrado entre m3 de agua por persona se ha mantenido bajo control, tal como se aprecia en la Tabla 1 :

En función de la Tabla 1, se puede decir que el ratio de consumo de agua por persona ha tendido a disminuir desde 0,43 en promedio los dos primeros años, a 0,31 en promedio en los dos últimos años de evaluación. Representando esto una reducción promedio de $28 \%$. En términos generales, desde el 2006 al 2014, se tiene un consumo promedio de $0.35 \mathrm{~m}^{3} /$ persona/año, lo cual es un logro importante para la entidad educativa en estos últimos nueve años, ya que la cantidad de personas se ha incrementado un $18 \%$, si comparamos los promedios de los 3 primeros y últimos años, del periodo de evaluación.

La verificación de los resultados a través del tiempo

\subsection{Sostenibilidad de la tendencia del uso del agua}

A partir de la estrategia desplegada para lograr el uso racional del agua, se planteó un modelo matemático que explique el consumo de agua por persona a partir de los registros de consumo de la entidad educativa.

$$
\text { Modelo: } \mathrm{Y}=\mathrm{Bo}+\mathrm{B} 1 \mathrm{X}
$$

En donde:

$$
\begin{aligned}
& \text { Y: variable dependiente } \\
& \mathrm{X} \text { : variable independiente }
\end{aligned}
$$

Este modelo se valida a partir del coeficiente de determinación $\left(R^{2}\right)$ y del análisis de varianza. Ambos análisis se realizaron a partir de la Tabla 2 .

En la Gráfica 2, se ilustra la línea de tendencia del consumo per-cápita de agua. Por un lado, el coeficiente de determinación $\left(R^{2}\right)$ es igual a 0,7049 e indica que el modelo es explicado hasta un $70,49 \%$, el cual es un porcentaje significativo. De otro lado, la pendiente de la ecuación es negativa, que denota la tendencia decreciente.

El valor $p$ del análisis de varianza es igual a 0,000, el mismo que permite rechazar Ho y afirmar que el modelo es significativo.

\subsection{Niveles de conciencia de los usuarios.}

Como resultado de la aplicación de una encuesta para medir el grado de conciencia de los usuarios sobre el uso del agua, se obtuvo que de las 240 personas entrevistadas un $58 \%$ considera que el agua debe ser utilizada de manera racional, otro $18 \%$ considera que debe ser restringido su uso. Si sumamos ambos grupos de personas obtenemos un $76 \%$ de respuestas a favor de la conservación del recurso hídrico. Esto se puede observar en la Gráfica 3 y 4. 
Tabla 1. Ratios de consumos $\mathrm{m}^{3}$ / persona - Año

\begin{tabular}{|c|c|c|c|}
\hline Año & $\mathrm{m}^{3}$ & Personas & $\mathrm{m}^{3} /$ persona \\
\hline 2006 & 113429 & 43357 & 0,38 \\
\hline 2007 & 91265 & 42408 & 0,46 \\
\hline 2008 & 89644 & 37569 & 0,42 \\
\hline 2009 & 113599 & 41539 & 0,37 \\
\hline 2010 & 159406 & 43834 & 0,27 \\
\hline 2011 & 154491 & 51382 & 0,33 \\
\hline 2012 & 189781 & 49513 & 0,26 \\
\hline 2013 & 160214 & 47475 & 0,30 \\
\hline 2014 & 148239 & 48396 & 0,33 \\
\hline
\end{tabular}

Fuente: Sistema integrado de gestión del SENATI

Tabla 2. Resultados por Semestre Académico

\begin{tabular}{|c|c|c|c|c|}
\hline Semestre & $\mathrm{m}^{3}$ & Personas & Persona/m3 & $\%^{*}$ \\
\hline 2006 I & 58983 & 22546 & 0,38 & $52 \%$ \\
\hline 2006 II & 54446 & 20811 & 0,38 & $48 \%$ \\
\hline 2007 I & 49304 & 23324 & 0,47 & $55 \%$ \\
\hline 2007 II & 40340 & 19084 & 0,47 & $45 \%$ \\
\hline 2008 I & 84485 & 19912 & 0,24 & $53 \%$ \\
\hline 2008 II & 74921 & 17657 & 0,24 & $47 \%$ \\
\hline 2009 I & 91095 & 19939 & 0,22 & $48 \%$ \\
\hline 2009 II & 98686 & 21600 & 0,22 & $52 \%$ \\
\hline 2010 I & 75602 & 22355 & 0,30 & $51 \%$ \\
\hline 2010 II & 72637 & 21479 & 0,30 & $49 \%$ \\
\hline 2011 I & 61000 & 20288 & 0,33 & $39 \%$ \\
\hline 2011 II & 93490 & 31094 & 0,33 & $61 \%$ \\
\hline 2012 I & 93574 & 24413 & 0,26 & $49 \%$ \\
\hline 2012 II & 96207 & 25100 & 0,26 & $51 \%$ \\
\hline 2013 I & 84548 & 25053 & 0,30 & $53 \%$ \\
\hline 2013 II & 75666 & 22422 & 0,30 & $47 \%$ \\
\hline 2014 I & 84882 & 27712 & 0,33 & $57 \%$ \\
\hline 2014 II & 63357 & 20684 & 0,33 & $43 \%$ \\
\hline
\end{tabular}

Fuente: Elaboración propia a partir de Estadísticas SENATI

* Porcentaje de consumo de agua y personas por semestre.

Análisis de Varianza:

Ho: la pendiente del modelo es nula

$\mathrm{H} 1$ : la pendiente del modelo no es nula 
Gráfica 2. Tendencia del Consumo

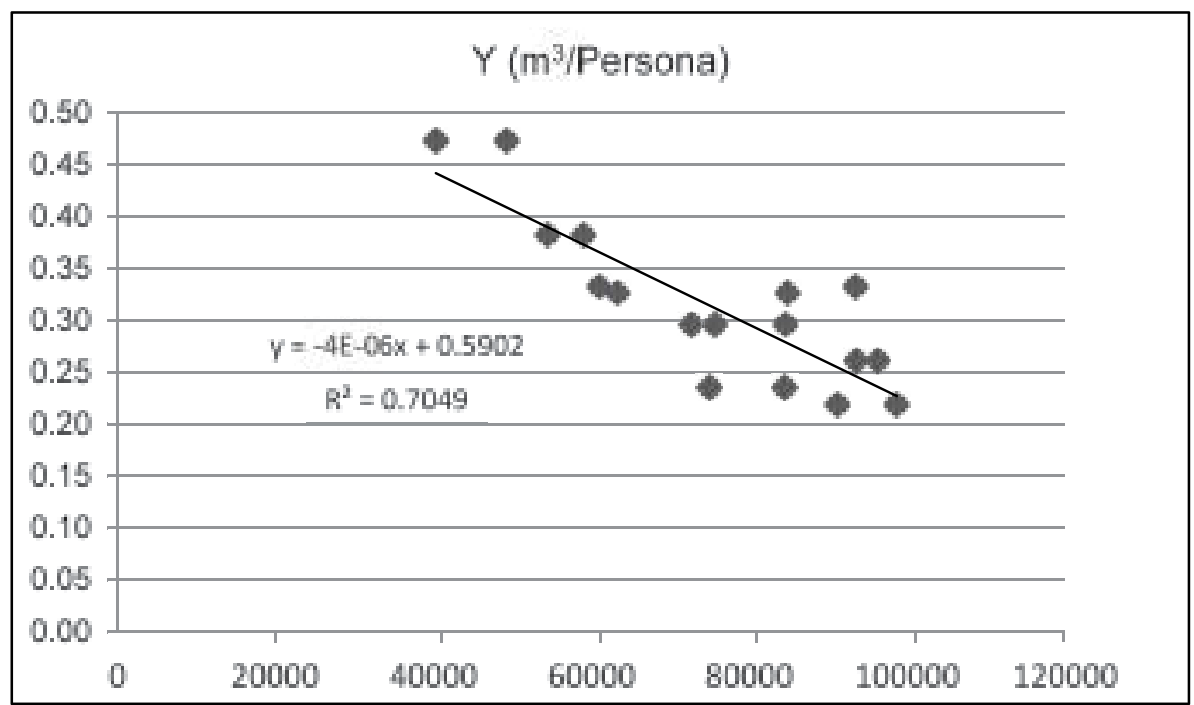

Tabla 3. Análisis de la Varianza del modelo de regresióna

\begin{tabular}{|c|c|c|c|c|c|c|}
\hline \multicolumn{2}{|c|}{ Modelo } & $\begin{array}{c}\text { Suma de } \\
\text { cuadrados }\end{array}$ & $\begin{array}{c}\text { Grados de } \\
\text { Libertad }\end{array}$ & $\begin{array}{c}\text { Media } \\
\text { cuadrática }\end{array}$ & F & Sig. \\
\hline \multirow{3}{*}{1} & Regresión & 668514 & 1 & 668514 & 39922 &, $000 \mathrm{~b}$ \\
\cline { 2 - 8 } & Residual & 267931 & 16 & 16746 & & \\
\cline { 2 - 8 } & Total & 936444 & 17 & & & \\
\hline
\end{tabular}

a. Variable dependiente: $y$

b. Variables predictoras: (Constante), $\mathrm{x}$

\section{CONCLUSIONES}

1. Para optimizar el uso del agua en una entidad educativa, es necesario aplicar una estrategia basada en tres aspectos importantes: mejorar los niveles de conciencia ambiental de los usuarios, Implementar mecanismos de control y establecer lineamientos claros relacionados y orientados al cuidado de dicho recurso.

2. El modelo de regresión encontrado revela la tendencia decreciente del consumo de agua por persona, tendencia que es significativa.

3. Es evidente que dentro de todas las variables que permiten utilizar racionalmente el agua, es el nivel de conciencia ambiental aquella que apuntala de mejor manera cualquier esfuerzo orientado al uso racional del agua. Esto se evidenció cuando se analizaron los resultados de los dos primeros años de aplicada la estrategia (2007 y 2008), años en los cuales se lograron los mejores resultados.
4. La aplicación de la encuesta permitió concluir que la mayoría de personas desea evitar desperdicios y cambiar malas prácticas 0 hábitos para utilizar racionalmente el agua.

5. Muchos opinan que aquellos que no cuidan el agua lo hacen porque presumen que el agua es un recurso inagotable. Esta opinión permite validar que existe un gran nivel de conciencia sobre el recurso para la mayoría de entrevistados.

6. EI SENATI, es reconocido como una entidad que busca cuidar el ambiente a través del uso racional del agua, específicamente a través de la educación ambiental de los alumnos y trabajadores.

7. Los mecanismos de control establecidos en el SENATI, tales como las inspecciones realizadas a las instalaciones sanitarias, son efectivos para contribuir con el uso racional del agua. Estas inspecciones aportan evitando los desperdicios 
Gráfica 3. Nivel de Conciencia de los usuarios

\section{P2.- ¿Cómo considera Ud. que se debe usar el agua?}

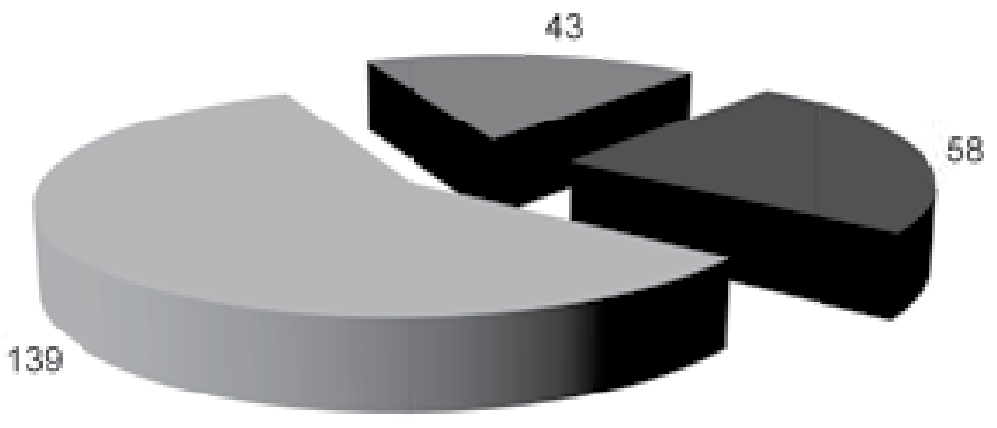

aa. Libremente. b. De forma racional ac. Restringiendo su uso.

Gráfica 4. Importancia del agua para los usuarios

P3.- ¿Cuál es la importancia de utilizar el agua de manera racional?

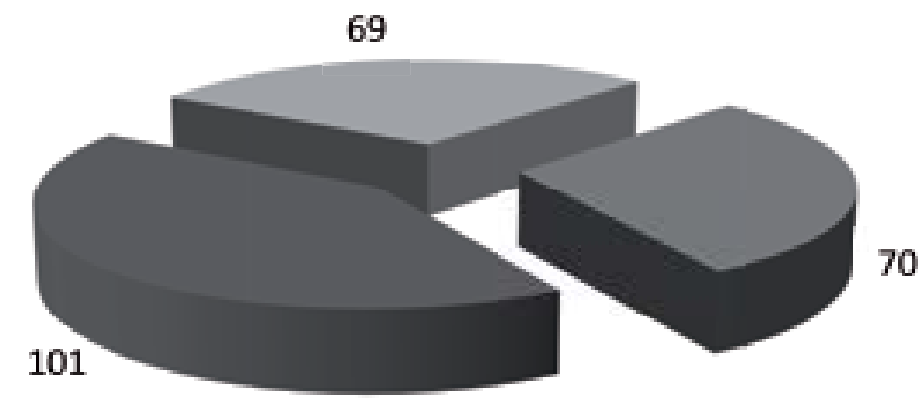

a. El agua estará disponible por mucho tiempo.

b. Ahorraremos dinero.

c. Muchas más personas podrán utilizarla.

propios del sistema de distribución de agua, tales como la detección, reporte de fugas, las cuales son reparadas a través de mantenimientos correctivos.

8. La entidad educativa, requiere urgentemente modificar el sistema de riego de áreas verdes, dado que los métodos actuales, si bien es cierto buscan evitar desperdicios no obedecen a una tecnología actualizada que sea más eficiente en la distribución del agua.

\section{RECOMENDACIONES}

1. Resultaría conveniente para la entidad educativa implementar sistemas más efectivos que le permitan utilizar el agua de manera más racional. Por ejemplo, instalar un sistema de riego de aspersores emergentes en las principales áreas verdes, en especial en su campo de fútbol.

2. Adicionalmente, sería interesante que instalen sistemas de captación de aguas grises (duchas y lavaderos) en una red de cisternas enterradas, 
con la finalidad de darle un tratamiento físico-químico a dichas aguas grises y luego reutilizarlas en el riego de las áreas verdes. Esta práctica es ampliamente conocida y aplicada en muchas empresas y organizaciones.

3. Incorporar dentro de sus planes de desarrollo de remodelaciones o nuevos edificios, tecnologías limpias que incluyan el ahorro de agua a través de la instalación de por ejemplo: griferías ahorradoras, tuberías con dispositivos de control de fugas, medidores y contómetros sectorizados, captación de aguas grises, aspersores giratorios, etc.

4. Seguir en la búsqueda de optimizar aún más el promedio de $3,5 \mathrm{~m}^{3} /$ persona/año obtenido en los últimos años.

5. Difundir a través de diversos canales de comunicación, los logros ambientales que han conseguido hasta la fecha, de tal manera que la sociedad perciba no solo que es una entidad de excelencia en adiestramiento en trabajo industrial y actividades afines, sino también comprometida responsablemente con la sostenibilidad de sus actividades.

\section{REFERENCIAS BIBLIOGRÁFICAS}

[1] López Badilla, G. (2006). Sistema de ahorro de agua doméstico con equipos electrónicos. México: CONALEP.

[2] Winn M. y Angell L. (2000). Towards a Process Model of Corporate Greening. Canadá: Faculty of Business, University of Victoria.

[3] Handfield, R.B., Walton, S.V., Seegers, L.K., y Melnyk. S.A. (1997). "Green" value chain practices in the furniture industry. Journal of Operations Management, 15 (3), pp 293-315.

[4]. Hutchinson, C. (1996). Integrating environmental policy with business strategy. Long Range Planning, pp 11-23.

[5] Gupta, M.C. y Sharma, K. (1996). Environmental operations management: an opportunity for improvement. Production and Inventory Management Journal, 37 (3), pp 40-46.

[6] Azzone G. y Noci G., (1998). Identifying effective PMS's for the deployment of "green" manufacturing strategies. International Journal of Operations \& Production Management, 18(4), 308-335. 